\title{
Does mRNA SARS-CoV-2 vaccine influence patients' performance during IVF-ET cycle?
}

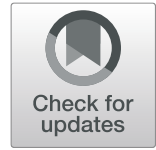

Raoul Orvieto ${ }^{1,2,3^{*}}$ D, Meirav Noach-Hirsh ${ }^{1,2}$, Aliza Segev-Zahav ${ }^{1,2}$, Jigal Haas ${ }^{1,2}$, Ravit Nahum ${ }^{1,2}$ and Adva Aizer ${ }^{1,2}$

\begin{abstract}
Objective: No information exists in the literature regarding the effect of mRNA SARS-CoV-2 vaccine on subsequent IVF cycle attempt. We therefore aim to assess the influence of mRNA SARS-CoV-2 vaccine on IVF treatments.

Design: An observational study.

Setting: A tertiary, university-affiliated medical center.

Patients and Methods: All couples undergoing consecutive ovarian stimulation cycles for IVF before and after receiving mRNA SARS-CoV-2 vaccine, and reached the ovum pick-up (OPU) stage. The stimulation characteristics and embryological variables of couples undergoing IVF treatments after receiving mRNA SARS-CoV-2 vaccine were assessed and compared to their IVF cycles prior to vaccination.

Main outcome measures: Stimulation characteristics and embryological variables.

Results: Thirty-six couples resumed IVF treatment 7-85 days after receiving mRNA SARS-CoV-2 vaccine. No inbetween cycles differences were observed in ovarian stimulation and embryological variables before and after receiving mRNA SARS-CoV-2 vaccination.
\end{abstract}

Conclusions: mRNA SARS-CoV-2 vaccine did not affect patients' performance or ovarian reserve in their immediate subsequent IVF cycle. Future larger studies with longer follow-up will be needed to validate our observations.

Keywords: COVID-19, vaccination, Ovarian stimulation, embryo quality, IVF

\section{Introduction}

Coronavirus disease 19 (COVID-19) emerged in Wuhan, Hubei province, China [1] in December 2019, and rapidly spread worldwide, affecting millions of people, with more fatalities compared with the SARS and MERS coronavirus epidemics combined.

When considering the relationship between COVID19 infection and infertility or infertility treatments, the ASRM Coronavirus/COVID-19 Task Force [2] emphasized that the existing evidence suggests that "the virus likely does not infect gametes [3, 4] or embryos",

\footnotetext{
* Correspondence: raoul.orvieto@sheba.health.gov.il

${ }^{1}$ Department of Obstetrics and Gynecology, Chaim Sheba Medical Center

(Tel Hashomer), Ramat Gan, Israel

${ }^{2}$ Sackler Faculty of Medicine, Tel Aviv University, Tel Aviv, Israel

Full list of author information is available at the end of the article
}

although no information exists in the literature regarding the influence of COVID-19 infection on laboratory/ embryological variables nor ovarian stimulation (OS) during the subsequent in-vitro fertilization (IVF) cycle attempt- which is considered the " most reliable sign of decrease ovarian reserve" [5].

Recently, we assessed the influence of COVID-19 infection on the stimulation characteristics and embryological variables of patients' IVF treatments, before and after recovering from COVID-19 infection [6]. COVID19 infection did not affect patients' performance or ovarian reserve in their immediate subsequent IVF cycle, except for a reduced proportion of top quality embryos (TQEs).

The newly available mRNA SARS-CoV-2 vaccine by Pfizer has been shown to be $95 \%$ effective in preventing 
SARS-CoV-2 infection a week following the second dose, with a favorable safety profile in a 2-month median follow up time [7]. It was shown to elicit high SARSCOV2 neutralizing antibody titers alongside high antigen specific CD8 + and Th1 type CD4+ T cell response. Prompted by the aforementioned observations, unfounded claims in the popular media linked a possible correlation between the SARS-CoV-2 vaccine and potential infertility. Currently, there is no information in the medical literature to confirm or dispute these unfounded claims. The aim of this observational study was to investigate the effect of BNT162b2 SARS-CoV-2 vaccination on OS characteristics and the embryological variables during the IVF treatment post COVID-19 infection, in order to aid both fertility specialists counselling and their patients in their decision-making process.

\section{Patients and methods}

The study population consisted of all couples undergoing consecutive OS for IVF, before and following the second dose of the vaccination, and reached the ovum pick-up (OPU) stage. The study was approved by the institutional research ethics board of Sheba Medical Centre.

Data on patient age and infertility-treatment-related variables were collected from the files. Embryological/laboratory variables of the IVF cycles were assessed and compared between the patients' IVF cycle before and following the second dose of the vaccination. Embryos classification was based on the individual embryo scoring parameters according to pre-established definitions [8]. A TQE was defined as seven or more blastomeres on day 3, equally-sized blastomeres and $\leqq 10 \%$ fragmentation.

Following a positive pregnancy test, ongoing pregnancies were confirmed by presence of gestational sac with fetal heart rate on ultrasound at 6-8-week gestation.

Statistical analysis was performed with pairedStudent's t-test and Chi square, as appropriate. Results are presented as means \pm standard deviations; $\mathrm{p}<0.05$ was considered significant.

\section{Results}

Of all couples who underwent IVF cycle treatments in our centre before the COVID-19 pandemic, in 36, both partners received the two doses of the vaccination. None of the couples suffered from co-morbidities (e.g. diabetes, obesity, HTN, asthma, cardiac disease). The interval between the time of the second vaccine to the date of the subsequent IVF treatment cycle was 7-85 days.

Patients clinical characteristics and the details of their IVF cycle attempts, before and after the mRNA SARSCoV-2 vaccine, are shown in Tables 1 and 2 . There were no differences between the cycles in the length of OS,

Table 1 Patients' baseline clinical characteristics

\begin{tabular}{|c|c|c|}
\hline & Female & Male \\
\hline Number of patients & 36 & 36 \\
\hline Mean interval between OPU cycles / Sperm test (months) & $5.2 \pm 6.1$ & $4.2 \pm 4.9$ \\
\hline (Range) & $(1-24)$ & $(0-23)$ \\
\hline Mean interval between Second Vaccination to OPU cycle / Sperm test (days) & $32.6 \pm 17.5$ & $33.3 \pm 14.9$ \\
\hline (Range) & $(7-85)$ & $(7-85)$ \\
\hline Age, years (mean $\pm S D$ ) & $37.3 \pm 4.6$ & $40.1 \pm 4.8$ \\
\hline Gravida (mean \pm SD) & $2 \pm 1.5$ & --- \\
\hline Para $($ mean $\pm S D)$ & $0.9 \pm 0.9$ & --- \\
\hline Smoking (\%) & $3 / 36(8.3)$ & --- \\
\hline $\mathrm{BMI}, \mathrm{Kg} / \mathrm{m}^{2}$ (mean $\left.\pm \mathrm{SD}\right)$ & $26.3 \pm 5.4$ & --- \\
\hline Mean basal FSH IU/L (mean \pm SD) & $8.2 \pm 3.8$ & --- \\
\hline Mean basal LH IU/L (mean \pm SD) & $5.1 \pm 2.5$ & --- \\
\hline \multicolumn{3}{|l|}{ Type of infertility } \\
\hline Male (\%) & $6 / 36(16.7)$ & \\
\hline Tubal (\%) & $1 / 36(2.8)$ & \\
\hline Endometriosis (\%) & 4/36 (11.1) & \\
\hline Unexplained infertility (\%) & $4 / 36(11.1)$ & \\
\hline Ovulatory disorder (\%) & $1 / 36(2.8)$ & \\
\hline Uterine factor (\%) & $1 / 36(2.8)$ & \\
\hline Others (\%) & $26 / 36(72.2)$ & \\
\hline
\end{tabular}


Table 2 Patients' OS variables and IVF cycle laboratory characteristics Pre/Post Covid-19 Vaccination

\begin{tabular}{|c|c|c|c|}
\hline & Pre Vaccination & Post Vaccination & $P$-value \\
\hline Antagonist Protocol (\%) & $34 / 36(94.4)$ & $35 / 36(97.2)$ & NS \\
\hline Duration of stimulation (days) & $10.7 \pm 2.6$ & $10.8 \pm 2.2$ & NS \\
\hline Total FSH dose used, IU (mean \pm SD) & $3802 \pm 1956$ & $3906 \pm 1565$ & NS \\
\hline Mean peak estradiol levels pmol/L (mean \pm SD) & $6041 \pm 4052$ & $7708 \pm 7640$ & NS \\
\hline Mean peak progesterone levels nmol/L (mean \pm SD) & $2.3 \pm 1.8$ & $2.2 \pm 1.2$ & NS \\
\hline Mean \# of Oocytes per OPU (mean \pm SD) & $9.7 \pm 6.7$ & $10.1 \pm 8$ & NS \\
\hline Mean \# of MII per OPU (mean \pm SD) & $7.94 \pm 5.7$ & $8.0 \pm 6.5$ & NS \\
\hline Mean \# of MII / \# of oocytes retrieved (mean \pm SD) & $0.83 \pm 0.1$ & $0.78 \pm 0.2$ & NS \\
\hline Mean \# of 2PN per OPU (mean \pm SD) & $6.44 \pm 5.0$ & $6.5 \pm 5.8$ & NS \\
\hline Mean \# of 2PN/ \# of oocytes retrieved (mean \pm SD) & $0.69 \pm 0.2$ & $0.63 \pm 0.2$ & NS \\
\hline Mean \# of TQE per OPU (mean \pm SD) & $2.8 \pm 2.7$ & $2.8 \pm 3.3$ & NS \\
\hline Mean \# of TQE/ \# of 2PN(mean \pm SD) & $0.40 \pm 0.3$ & $0.40 \pm 0.2$ & NS \\
\hline Semen volume $(\mathrm{mL})($ mean $\pm \mathrm{SD})$ & $3.0 \pm 1.4$ & $3.2 \pm 1.7$ & NS \\
\hline Sperm concentration (Millions)(mean \pm SD) & $72.4 \pm 61.5$ & $80.2 \pm 55.7$ & NS \\
\hline Sperm \% motility (mean \pm SD) & $56 \pm 22$ & $54 \pm 20$ & NS \\
\hline Pre-wash total motile sperm count, millions (mean \pm SD) & $134 \pm 169$ & $146 \pm 159$ & NS \\
\hline
\end{tabular}

total dose of gonadotropin used, nor the peak estradiol and progesterone levels (Table 2).

Furthermore, no differences were observed in the number of oocytes and mature oocytes retrieved, fertilization rate, TQE and the ratio of TQEs per number of $2 \mathrm{PN}$, or semen analyses (Table 2).

No patients conceived in the IVF treatment cycle before receiving the vaccine, while 3 pregnancies were recorded in the 10 patients who underwent embryo transfer (30\% per transfer) in the cycle following mRNA SARS-CoV-2 vaccination.

\section{Discussion}

In the present study we observed no influence of mRNA SARS-CoV-2 vaccine on patients' performance during their immediate subsequent IVF cycle, reflecting no detrimental effects of the vaccine on patients' ovarian reserve, nor the developing gametes/embryos, with an acceptable pregnancy rate ( $30 \%$ per transfer).

To date, damage to the female reproductive system in COVID-19 patients has not been reported. There is indirect evidence that COVID-19 might affect female fertility by attacking ovarian tissue and granulosa cells, and decreasing ovarian function and oocyte quality. Moreover, COVID-19 might damage endometrial epithelial cells and affect early embryo implantation [9-12]. A recent study by our group could not demonstrate any effect of COVID-19 infection on the OS characteristics and embryological variables of patients' IVF treatments, except for a reduced proportion of top quality embryos.

Since folliculogenesis and spermatogenesis are complex and dynamic processes involving multiple endocrine cells and numerous signals that have been estimated to span $>3$ months $[13,14]$. The COVID-19 infection, by its known ability to activate the release large amounts of pro-inflammatory cytokines and precipitate and sustain an aberrant systemic inflammation [15], might also interfere with these processes, resulting in abnormal gametes (oocytes and sperms), with the consequent production of low quality embryos.

Following mRNA SARS-CoV-2 vaccine, we could not observe any detrimental effect on OS characteristics, embryological variables nor the proportion of top quality embryos. These might be explained by the lesser degree of systemic inflammation induced by the vaccine, with modest effect on folliculogenesis and spermatogenesis.

In the present study, we could not demonstrate any detrimental effect of mRNA SARS-CoV-2 vaccine on ovarian reserve/ oocytes pool, as reflected by the similar response to OS- which is considered the " most reliable sign of decrease ovarian reserve [5]. Moreover, since the IVF treatment attempts were conducted 7-85 days post vaccination, when the retrieved gametes during these cycles were exposed to the mRNA SARS-CoV-2 vaccine induced systemic inflammation during their development, in contrast with active infection [6], any potential inflammatory environment following the vaccine did not interfere with the intricate complex processes of folliculogenesis and spermatogenesis.

Regarding the effect of COVID-19 on the male reproductive system, this issue is even more controversial. While 5 studies failed to detect the presence of COVID19 viral RNA in the semen samples of patients with active or resolving infection [16-20], one study identified 
COVID-19 RNA in $15.38 \%$ of the semen samples [21] and another study [20] demonstrated that patients with moderate infection had significantly reduced sperm quantity and quality, compared to patients with mild infection or normal controls. In the present study, mRNA SARS-CoV-2 vaccine showed no detrimental effect on patients' total motile count.

The limitations of our study is the small sample size and the short period of follow-up. A major strength of our study is that we compared two consecutive IVF cycle attempts (before and following vaccination) in the same cohort of patients. The fact that all women that participated in our study had two consecutive treatment cycles, helps to eliminate multiple bias factors and to attribute the study results to the pre and post vaccination effect.

In conclusions, mRNA SARS-CoV-2 vaccine did not affect patients' performance or ovarian reserve in their immediate subsequent IVF cycle. Future larger studies with longer follow-up will be needed to validate our observations.

\section{Abbreviations}

COVID-19: Coronavirus disease 19; IVF: In-vitro fertilization.; OPU: Ovum pickup.; OS: Ovarian stimulation.; TQE: Top quality embryos.

\section{Acknowledgements}

The authors would like to thank Dr Arie Yeshaya (Maccabi health care) for his contributions to Data collection.

\section{Authors' contributions}

R.O. Designed the study, wrote the first paper draft, edited it, proof read the paper and took part in discussions regarding the results. M.N.H. Retrieved the data, proof read the paper and took part in discussions regarding the results. A.S.Z., J.H., R.N., Proof read the paper and took part in discussions regarding the results. A.A. Designed the study, conducted the embryological work and retrieved the data, made the statistical analysis, proof read the paper and took part in discussions regarding the results. The author(s) read and approved the final manuscript.

\section{Funding}

N/A.

\section{Availability of data and materials}

N/A

\section{Declarations}

Ethics approval and consent to participate

The study was approved by our institutional review board (SMC-7901-20).

\section{Consent for publication}

Authors have nothing to declare.

\section{Competing interests}

Authors have nothing to declare.

\section{Author details}

${ }^{1}$ Department of Obstetrics and Gynecology, Chaim Sheba Medical Center (Tel Hashomer), Ramat Gan, Israel. ${ }^{2}$ Sackler Faculty of Medicine, Tel Aviv University, Tel Aviv, Israel. ${ }^{3}$ The Tarnesby-Tarnowski Chair for Family Planning and Fertility Regulation, Sackler Faculty of Medicine, Tel-Aviv University, Tel Aviv, Israel.
Received: 22 April 2021 Accepted: 6 May 2021

Published online: 13 May 2021

\section{References}

1. Huang C, Wang Y, Li X, Ren L, Zhao J, Hu Y, et al. Clinical features of patients infected with 2019 novel coronavirus in Wuhan, China. Lancet. 2020;395(10223):497-506.

2. ASRM patient management and clinical recommendations during the CORONAVIRUS (COVID-19) pandemic Update No. 10 (November 17,2020). https://www.asrm.org/globalassets/asrm/asrm-content/news-andpublica tions/covid-19/covidtaskforceupdate10.pdfâ.

3. Barragan M, Guillén JJ, Martin-Palomino N, Rodriguez A, Vassena R. Undetectable viral RNA in oocytes from SARS-CoV-2 positive women. Hum Reprod Sep. 2020;30:deaa284. https://doi.org/10.1093/humrep/deaa284. Epub ahead of print. PMID: 32998162

4. Ruan Y, Hu B, Liu Z, Liu K, Jiang H, Li H, Li R, Luan Y, Liu X, Yu G, Xu S, Yuan X, Wang S, Yang W, Ye Z, Liu J, Wang T. (2020) No Detection of SARS-CoV-2 from Urine, Expressed Prostatic Secretions and Semen in 74 Recovered COVID-19 Male Patients: A Perspective and Urogenital Evaluation. Andrology. Nov 4. https://doi.org/10.1111/andr.12939. Epub ahead of print. PMID: 33150723.

5. Gleicher N, Barad D. Unexplained infertility: Does it really exist? Hum Reprod. 2006;21:1951-530.

6. Orvieto R, Segev-Zahav A, Aizer A. Does COVID-19 infection influence patients' performance during IVF-ET cycle?: an observational study. Gynecol Endocrinol 2021. (in press).

7. Polack FP, Thomas SJ, Kitchin N, et al. Safety and Efficacy of the BNT162b2 mRNA Covid-19 Vaccine. N Engl J Med. 2020;383(27):2603-15.

8. Ziebe S, Lundin K, Janssens R, Helmgaard L, Arce J-C, for the MERIT Group. Influence of ovarian stimulation with HP-hMG or recombinant FSH on embryo quality parameters in patients undergoing IVF. Hum Reprod. 2007;22:2404-13.

9. Reis FM, Bouissou DR, Pereira VM, Camargos AF, Dos Reis AM, Santos RA. Angiotensin-(1-7), its receptor Mas, and the angiotensin-converting enzyme type 2 are expressed in the human ovary. Fertil Steril. 2011;95:176-81.

10. Vaz-Silva J, Carneiro MM, Ferreira MC, Pinheiro SVB, Silva DA, Silva AL, Witz CA, Reis AM, Santos RA, Reis FM. The Vasoactive Peptide Angiotensin-(1-7), Its Receptor Mas and the Angiotensin-converting Enzyme Type 2 are Expressed in the Human Endometrium. Reprod Sci. 2009;16:247-56.

11. Barreta $M H$, Gasperin BG, Ferreira R, Rovani M, Pereira GR, Bohrer RC, De Oliveira JF, Gonçalves PBD. The components of the angiotensin-(1-7) system are differentially expressed during follicular wave in cattle. J Renin Angiotensin Aldosterone Syst. 2013;16:275-83.

12. Pereira VM, Reis FM, Santos RA, Cassali GD, Santos SH, Honorato-Sampaio K, Dos Reis AM. Gonadotropin stimulation increases the expression of angiotensin-(1-7) and MAS receptor in the rat ovary. Reprod Sci. 2009;16(12):1165-74.

13. Gougeon A. Dynamics of follicular growth in the human: a model from preliminary results. Hum Reprod. 1986;1:81-7.

14. de Kretser DM, Loveland KL, Meinhardt A, Simorangkir D, Wreford N. Spermatogenesis Hum Reprod. 1998;13(1):1-8.

15. Coperchini F, Chiovato L, Croce L, Magri F, Rotondi M. The cytokine storm in COVID-19: An overview of the involvement of the chemokine/ chemokine-receptor system. Cytokine Growth Factor Rev. 2020;53:25-32.

16. Pan F, Xiao X, Guo J, Song Y, Li H, Patel DP, et al. No evidence of severe acute respiratory syndrome-coronavirus 2 in semen of males recovering from coronavirus disease 2019. Fertil Steril. 2020; 113:1135-9.

17. Song C, Wang Y, Li W, Hu B, Chen G, Xia P, et al. Absence of 2019 novel coronavirus in semen and testes of COVID-19 patients. Biol Reprod. 2020;103:4-6.

18. Paoli D, Pallotti F, Colangelo S, Basilico F, Mazzuti L, Turriziani O, et al. Study of SARS-CoV-2 in semen and urine samples of a volunteer with positive naso-pharyngeal swab. J Endocrinol Invest. 2020;43(12):1819-22.

19. Holtmann N, Edimiris P, Andree M, Doehmen C, Baston- Buest D, Adams O, et al. Assessment of SARS-CoV-2 in human semen-a cohort study. Fertil Steril. 2020;114(2):233-8

20. Ning J, Li W, Ruan Y, Xia Y, Wu X, Hu K, et al. Effects of 2019 novel coronavirus on male reproductive system: a retrospective study. Preprints. 2020. https://doi.org/10.20944/preprints202004. 0280.v1.

21. Li D, Jin M, Bao P, Zhao W, Zhang S. Clinical characteristics and results of semen tests among men with coronavirus disease 2019. JAMA Netw Open. 2020; 3:e208292

\section{Publisher's Note}

Springer Nature remains neutral with regard to jurisdictional claims in published maps and institutional affiliations. 